\title{
Digital dentistry: The future is now
}

\author{
Supreet K. Thind ${ }^{1, *}$, Shiva Gupta ${ }^{2}$, Rasveen Kaur ${ }^{3}$, Surinder Sachdeva ${ }^{4}$, Aastha Bakshi ${ }^{5}$ \\ ${ }^{1-3}$ Senior Lecturer, ${ }^{4}$ Professor and HOD, ${ }^{5}$ Post Graduate Student, Dept. of Periodontics, Maharishi Markandeshwar College of \\ Dental Sciences and Research, Mullana Ambala, Haryana, India
}

*Corresponding Author:

Email: dr.supreet.thind@gmail.com

\begin{abstract}
Dentistry has witnessed tremendous advances in all its branches over the past few decades. Computer technology is an essential ingredient for state-of-the-art patient care and is becoming an integral part of the practice of dentistry. Smaller, smarter computing devices will support an increasing proportion of dental practice activities. Technology will make practice management more efficient. Computer software which could easily inter-relate photographic images and esthetic and cosmetic measurements would be a dynamic clinical tool and it is also beneficial in research to be conducted for cosmetic and esthetic purpose. Digital dentistry is not the wave of the future; it is occurring now. Whether a dentist embraces new technology will define his or her practice and, possibly, future.
\end{abstract}

Keywords: Computers, Dental education, Dentistry, Computer assisted learning, Dental imaging.

\section{Introduction}

The digital revolution that is transforming every aspect of our day to day life is also impacting dentistry and medicine in a multiple ways, from electronic record-keeping and data analysis to new diagnostic tools, novel prevention methods and revolutionary treatment options. ${ }^{1}$ Patient and practice management softwares, web-based learning programs etc, have influenced the art and science of dentistry in recent times. Simplified interactions between human and computer have caused a profound progress in virtual reality (VR)-based dental training. On the other hand, computer-aided design/computer aided manufacturing (CAD/CAM) of dental appliances and prostheses is now widely used around the globe. ${ }^{2}$

\section{History ${ }^{3}$}

The use of digital computers in biomedicine traces its origin closely to the seminal events that mark the beginning of the computer revolution.

1820: Charles Babbage began his lifelong quest for a programmable machine, the analytical engine, recognized as the first general purpose computer. Known as the father of computing.

1842: Ada Lovelace used it to mechanically translate a short written work. Regarded as the first programmer.

1854: George Boole wrote An Investigation of the Laws of Thought, and recognized as the father of computer science.

1936: John Vincent Atanasoff, assisted by a student Clifford Berry invented a digital computer, the "ABC". It displays some early features of later computers including electronic calculations.

1941: Konrad Zuse developed first electromechanical digital computer

1946: Mauchly's and Eckert's Electronic Numerical Integrator and Calculator (ENIAC)
1949: A N Wang, the invention of the transistor at Bell Labs in 1948, and the development of electronic core memory.

\section{What is a computer?}

Any programmable electronic device that can store, retrieve and process data. (Webster's dictionary).

What is digital dentistry?

Digital dentistry may be defined in a broad scope as any dental technology or device that incorporates digital or computer-controlled components in contrast to that of mechanical or electrical alone.

What is dental informatics?

It is the "application of computer and information science to improve dental practice, research and program administration". ${ }^{4}$

\section{Digital Dentistry Applications}

The computers are used for many purposes in the field of dentistry and can be broadly classified as: ${ }^{5-7}$

1. Administrative applications

2. Clinical applications \&

3. Other applications

Administrative applications ${ }^{5-7}$

They are aimed for a smooth running of the dental clinics, the hospitals \& the dental institutions
a) Patient appointments and recalls
b) Billing
c) Accounting
d) Correspondence
e) Inventory control and supply orders
f) Dental insurance claims
g) Document preparation \& word processing
h) Referral information
i) Missed appointments follow-up

\section{Clinical Applications ${ }^{5-7}$}

The computers are very useful for the dentists in their professional practice: 
a) Patient record storage \& retrieval

b) Clinical diagnosis and treatment planning

c) Patient motivation \& awareness

d) Computerized cephalometrics \& growth prediction.

e) CAD-CAM

f) Computer assisted densitometer image analysis system

g) For special purposes like computerized spirometers, blood chemistry \& gas analysers, ultrasound scanners and CT scanners

h) Radiovisuography (RVG) technique

i) Storage of patient photographs, radiographs \& study models.

Other applications $^{5-7}$

a) Controlling the circulation of books and journals and their availability in the library.

b) Creating a data base of survey information.

c) Case presentation

d) Conference presentations

e) Reviewing of literature

f) Entertainment \& family use

g) Continuing dental education.

\section{A. Computerised Dental Patient Record ${ }^{8,9}$}

1. Electronic dental patient record (EDPR) is storing patient information in a digital format that can be retrieved, duplicated, cataloged and transmitted as needed.

2. Record storage becomes difficult as the practice ages, bulky paper records and study models pose a problem of organization and space.

3. Current technology is available for in office use of computers combined with video cameras to copy radiographs, study models, photographs and hand written records for storage in compact digital form.

4. With the EDPR there is no longer necessity to maintain a fussy paper appointment book so the new dentistry era can be better called as paperless dentistry.

\section{Advantages of EDPR ${ }^{8}$}

1. Providing accurate, up-to-date, and complete information about patients at the point of care

2. Time saving

3. Compactness, minimum storage space

4. Portability and durability

5. Simple and quick accessing

6. Instant hard copies can be produced

7. Ease of record transmission

8. Cost savings and fewer workplace inefficiencies through decreased paperwork, improved safety, reduced duplication of testing and improved health.

9. Helping providers more effectively diagnose patients, reduce medical errors and provide safer care.

10. Improving patient and provider interaction and communication as well as health care convenience.

11. Enabling safer and more reliable prescribing.
12. Helping promote legible, complete documentation and accurate, streamlined coding and billing.

13. Enhancing privacy and security of patient data.

14. Helping providers improve productivity and worklife balance.

\section{Possible Problems of EDPR ${ }^{8}$}

1. Confidentiality and security issues. Computerized records can be easily altered without a trace so cannot be admissible as evidence in a court of law.

2. Standardization of the capture procedure for the images of study models will have to be established to make such images acceptable for all purposes.

3. Equipments and software in common use are not necessarily compatible.

4. High startup costs

5. Substantial learning curve

6. Lack of standardized terminology, system architecture, and indexing.

\section{B. Computers in Forensic Dentistry ${ }^{10-11}$}

The use of computers in forensic dentistry has mirrored the use of computers in dentistry in general and has presented new tools for solving difficult forensic problems thus creating new concerns regarding their application in general dentistry. ${ }^{10}$ Several research studies with application of software technology to identify an individual has been proposed and found to give very reliable results. Can be use to identify:

1. Palatal rugae pattern

2. Facial reconstruction

3. Maxillary sinus in gender determination:

4. Bite marks

5. Personal identification based on specific patterns of DMFS

Computer programs used for identification: Computer assisted postmortem identification system (CAPMI) tabulates restorations and missing teeth as sorting parameters.

\section{Computerized Dental Anxiety Scale for Children}

The Smiley Faces Program (SFP) is a four item computerised trait dental anxiety scale, using faces as a response set, to assess dental anxiety in children. Hence, faces have been used as a response set within this trait anxiety scale. ${ }^{12}$

Advantages: ${ }^{12}$

1. It is short in length so should maximize the responses by the children and minimize the time for administration.

2. It includes items that are relevant to most children's dental experience (eg having a tooth drilled, sitting in the dental waiting room).

3. The use of computer graphics should help the child engage with the dental anxiety scale.

4. Computer graphics offer interactive animations, which allow participants to control aspects of the visual display in a trial 
5. The program has the added advantage of a computer database with anxiety scores for immediate investigator access.

Some investigators have used computer-based questionnaires to see the effects of computer versus paper-and pencil administration on different types of measures. Children especially rate these as enjoyable, and see the computer version as preferable to pen and paper alternatives. ${ }^{13}$

\section{Computers in Research}

Commonly used software systems available for statistics, sample size calculation and power analysis are:

Statistical Package for the Social Sciences (SPSS manufactured by IBM corporation), Statistical Analysis System ((SAS - developed by SAS Institute North Carolina, United States of America), R (designed by Ross Ihaka and Robert Gentleman from R core team), Minitab (developed by Minitab Inc), Stata (developed by StataCorp) and the MS Excel (developed by Microsoft). ${ }^{14,30}$

There are a number of web resources which are related to statistical power analysis.

1. StatPages.net - provides links to a number of online power calculators

2. G-Power - provides a downloadable power analysis program that runs under DOS

3. Power analysis for ANOVA designs an interactive site that calculates power or sample size needed to attain a given power for one effect in a factorial ANOVA design

4. SPSS makes a program called Sample Power. It gives an output of a complete report on the computer screen which can be cut and paste into another document. ${ }^{14}$

\section{E. Computers for Patient Education ${ }^{15}$}

Dental software programs can provide patients with a $3 \mathrm{D}$ animation of their own teeth so as to explain them the treatment they require. Dental software programs also include a stock of images and educational shorts on procedures and recommendations for treatment. Educational leaflets, or pamphlets that provide patients with information regarding procedures they may be curious about or require, are helpful in preparing patients for the procedure and answering questions that they may have. Patient images can also be included with treatment plan letters to patients or as a marketing device in encouraging patients to accept treatment. Softwares like Microsoft office and PowerPoint are effectively used in continuing dental education programs.

INTRAORAL CAMERAS: They provide an empowering technology for diagnosis, patient education, case presentation, and virtual documentation. ${ }^{15}$

\section{F. Computerized Diagnosis}

Pocket probing is the crucial and mandatory procedure in diagnosing periodontitis and evaluating periodontal therapy.

\section{i) Computerized periodontal probes- ${ }^{16}$}

\section{Advantages:}

1. Very accurate readings

2. Standard force

3. Standard probe tip diameter

4. Convenience of digital readout and computer data storage

Drawbacks:

1. Expense

2. Extra time required

3. Difficult to use in posterior region of mouth or lingual surface of anterior teeth with long clinical crowns

Florida probe system: By Gibbs et al, 1988

Based on NIDCR criteria for overcoming the limitations of conventional periodontal probing:

National Institute of Craniofacial Research (NIDCR) defined nine criteria for overcoming limitations of conventional periodontal probing.

1. Precision: $0.1 \mathrm{~mm}$

2. Range: $10 \mathrm{~mm}$

3. Probing force: constant and standard

4. Applicability: non-invasive, light weight, and easy to use

Table 1:

\begin{tabular}{|l|c|c|}
\hline Limitation & $\begin{array}{c}\text { Conventional } \\
\text { Probing }\end{array}$ & $\begin{array}{c}\text { NIDCR } \\
\text { Criteria }\end{array}$ \\
\hline Precision & $1 \mathrm{~mm}$ & $0.1 \mathrm{~mm}$ \\
\hline Range & $12 \mathrm{~mm}$ & $10 \mathrm{~mm}$ \\
\hline Probing force & Nonstandardized & $\begin{array}{c}\text { Constant and } \\
\text { standardized }\end{array}$ \\
\hline Angulation & Subjective & $\begin{array}{c}\text { Guidance } \\
\text { system to ensure } \\
\text { proper } \\
\text { angulation }\end{array}$ \\
\hline Readout & $\begin{array}{c}\text { Depending on } \\
\text { voice dictation } \\
\text { and recording in } \\
\text { writing }\end{array}$ & $\begin{array}{c}\text { Direct electronic } \\
\text { reading and } \\
\text { digital output }\end{array}$ \\
\hline
\end{tabular}

\section{Parts:}

1. probe handpiece

2. Digital readout

3. Foot switch

4. Computer interface and computer

5. Probe tip $(0.4 \mathrm{~mm}$ diameter $)$ reciprocates through a sleeve whose edge provides a reference by which measurements are made.

Types:

1. Pocket depth probe

2. Disk probe: record clinical attachment level relative to occlusal surface of teeth

3. Stent probe: record CAL relative to prefabricated stent. 


\section{Associated Problems:}

1. Lack tactile sensitivity because of independent movement of probing elements forcing the operator to predetermine insertion point and angle.

2. Fixed force setting throughout the mouth, regardless of the site or inflammatory status, may generate inaccurate measurements (underestimation has been reported by Perry et al.) or patient discomfort.

3. The Florida Probe provides precise electronic measurement to $+0.1 \mathrm{~mm}$ accuracy.

4. Considered as current gold standard for computerized probes.

The Foster-Miller probe (an electronic probe described by Jeffcoat et al 1986)

It is capable of coupling pocket depth measurement with the detection of cementoenamel junction from which clinical attachment level is automatically detected.

\section{The Toronto Automatic Probe}

1. Like florida probe uses the occusal incisal surface to measure the relative clinical attachment level.

2. The sulcus is probed with $0.5 \mathrm{~mm}$ nickel-titanium wire that is extended under pressure.

3. It controls angular discrepancies by means of a mercury tilt sensor that limits angulation within +/_ 30 degrees but it requires reproducible positioning of the patients head and cannot easily measure second and third molars

\section{ii) Advances in Radiography}

\section{Digital Radiography- ${ }^{17,38}$}

Digital radiography enables the use of computerized images which can be stored, manipulated, and corrected for under and over exposure. Moreover there in important dose reduction obtained with this technique (between $1 / 3$ to $1 / 2$ ). There are many new \& existing developments such as the wireless sensors (both CCD/CMOS and PSP), caries diagnosis (Logicon by Carestream Dental), Intelligent Positioning System for quick \& easy digital alignment of the tube head to the sensor (Carestream Dental) and integration with the tablets \& voice activation.

Two digital radiographic methods:

The direct method:

1. It uses a charged coupled device (CCD) sensor linked with the fiber optic or other wire to the computer system.

2. Obtains real time imaging offering improved visualisation of periodontium.

3. The disadvantages of this technique are

4. Limited sensor area which is only large enough to depict one or two teeth.

5. And the rigidity of the sensor

The Indirect Method: (Digora System)

1. Uses a phosphor luminescence plate, which is flexible film like radiation energy sensor placed intraorally and exposed to x-ray tube
2. A laser scanner reads the exposed plates offline and reveals digital image data.

Benefits of Digital Radiography:

1. Lower radiation (when following the ALARA principle),

2. Significant time reduction,

3. Ease of storage \& organization, and

4. The image enhancements for quick \& improved viewing

\section{Computer Assisted Densitrometric Image} Analysis System- ${ }^{18}$

Introduced by Urs Brägger et al 1988.

A video camera measures the light transmitted through a radiograph

Signals from camera are converted into gray scale images

Camera is interfaced with an image processor and a computer that allows storage and manipulation of images.

It offers an objective method for studying alveolar bone changes quantitatively. This gives a high degree of sensitivity, accuracy and reproducibility.

\section{Computer-based Thermal Imaging- ${ }^{19}$}

Aids in gingival temperature measurement. It compares the re-warming rates of normal and inflamed human gingiva. Valuable objective method for the diagnosis of periodontal diseases.

\section{Digital Image}

The electric signal that is produced by the sensor is a voltage that is varying as a function of time. The sensor is connected to a special board in the computer called a frame grabber; the function of this board is to sample the signal at short intervals, thus converting the analog signal into a digital signal. The output of the measurements is stored in the computer as numbers. ${ }^{20}$

When the image is captured and digitized by means of an electronic sensor system, the radiation intensities are measured a long a rectangular two- dimensional grid of sensor elements, called pixels. The outcome of the measurement of each sensor elements transferred to the computer and stored as a number between 0 and 255 . To display the image, the numbers are read out and used to control the intensities of the pixels on the monitor screen. Several methods exist to acquire a digital image. ${ }^{21}$

\section{Cone beam computed tomography}

CBCT developed in 1982 for angiography. Utilizes cone shaped source of ionizing radiation \& 2D area detector fixed on a rotating gantry. Multiple sequential images are produced in one scan. It rotates $360^{\circ}$ around the head. Scan time typically $<1$ minute. Image acquisition involves a rotational scan of a $\mathrm{x}$ ray source and reciprocating area detector moving synchronously around patients head. Many exposures are made at fixed intervals to form basic images. 
Software programs are used to reconstruct 3D images. ${ }^{22}$ It is indicated in evaluation of the jaw bones, Implant placement and evaluation of TMJ, Bony \& soft tissue lesions, periodontal assessment, endodontic assessment alveolar ridge resorption, airway assessment. It also aid in diagnosing periodontal pathologies like gingival hyperplasia, gingival recession, pathologies related to alveolar bone. ${ }^{23}$ Two dimensional imaging techniques are routinely used for the assessment of alveolar bone defects in periodontology.

\section{Denta CT scan}

Denta Scan is a unique computer software program provides computed tomographic (CT) imaging of the mandible and maxilla in three planes of reference: axial, panoramic, and oblique sagittal. Provides visualization of internal bone morphology in three dimensions; precise treatment planning. In cross sectional view, observation regarding bone quality, density can be made. Preoperative planning of endosseous dental implants and sub-periosteal implants. ${ }^{23}$ Dental scan CT provides information of the internal structures that cannot even be gained by direct intra-operative visualization, the bony structures visualization. It gives precise location of the mandibular canal and the floor of the maxillary sinuses.

\section{Advantages:}

Minimum additional cost;

Low radiation dose;

Multi planer reformation;

Eliminates treak artifacts;

Exact information about alveolar bone dimensions and Location of mandibular canal and maxillary sinus.

In implant imaging,it helps in measuring Bone quantity: Height and buccolingual dimension of implant site; Bone volume: Extent of bone resorption; Bone quality and precise location of vital structures. ${ }^{19}$

\section{iii) Optical Dental Diagnosis ${ }^{24}$}

1. Early diagnosis of dental caries is very important in initiating early treatment and preventing morbidity. Radiographs have not been really successful in this direction.

Recently, a confocal microscope is discovered, which is capable of use in the mouth. By utilizing low cost laser diodes and optical fibers the instrument is capable of recording depth profiles through a lesion. The resulting curves can then be analysed and the depth and state of de-mineralisation within the lesion determined.

In an alternative approach a low cost spectrometer system has been built for use in the oral cavity based around the latest generation of blue laser diodes. The $405 \mathrm{~nm}$ light excites fluorescence from within the tooth and this fluorescence is then spectroscopically analysed. By examining the relative emission from the correct wavelength regions it is possible to determine the status of the lesion and hence help with the diagnosis.

\section{G. Computers in Restorations ${ }^{17,25,38}$}

CAD/CAM technology was introduced to dentistry in 1988 in Germany and is widely used today to generate tooth-colored fillings. CAD/CAM is an acronym for computer- aided design/computer-aided manufacturing. With CAD/CAM, parts and components can be designed and machined with precision using a computer with integrated software linked to a milling device. $\mathrm{CAD} / \mathrm{CAM}$ can be used for making fillings chairside in the dental office or for fabricating restorations in a dental laboratory.

\section{Procedure:}

1. The dentist makes a digital picture of the prepared tooth with a small intraoral camera.

2. This digital image contains three-dimensional information about the size of the tooth and defect being restored, as well as the adjacent teeth.

3. The dentist will then design the desired filling directly on a computer screen using CAD/CAM software.

4. Once all of the pertinent information has been entered (such as type of filling, contours, etc.), a tooth-colored block of ceramic or composite material is machined by fine diamond drills to produce the designed filling. The CAD/CAM filling is then tried in the mouth, adjusted, polished, and bonded in place with a composite resin bonding cement.

Currently, there are more than eight companies that offer intraoral imaging, with CEREC (Sirona), E4D (D4D Technologies), LAVA COS (3M), and iTero (Cadent/Align) being the most recognized and used. CR Foundation (CLINICIANS REPORT) has researched all of these scanning systems and proven all to be as accurate as the conventional methods (i.e., stone die systems). Most are more accurate, faster, and easier.

\section{H. Computers in Occlusal Analysis ${ }^{26}$}

1. The T-scan II computerized occlusal analysis system allows a precise analyze your bite and identify any damaging occlusal forces. The ability to measure the force of a patient's bite is critical when it comes to assessing oral health and is an important parameter that determines periodontal and prosthodontic treatment.

2. It accurately detect forces on dental prostheses including fixed, fixed-removable dentures, complete removable dentures, bridges and even dental implants. It collects occlusal contact data that can be seen in clearly-defined graphics and printouts. This data can be combined with periodontal charting, X-ray and tissue exam data to provide a complete assessment of oral health. Tscan II is one of the best occlusal diagnostic tools available today. 


\section{Computer Controlled Anesthetic Delivery ${ }^{27}$}

The first computer controlled local anesthetic delivery (CCLAD) system was introduced into dentistry (wand/ compuDent) in 1997.

Similar systems:

QuickSleeper

Anaeject

Comfort Control Syringe

\section{Advantages:}

1. Enables the dentist to accurately manipulate needle placement with fingertip accuracy and deliver the LA with a foot activated control.

2. This provides quicker numbing of the teeth, without the usual numbness of the tongue, lips and face. Computer controlled anesthetic delivery also eliminates that "fat face feeling" that can last for hours.

3. Increased tactile sensation and control.

4. Pen grasp allows rotation of light weight hand piece minimizing the needle deflection and the force for penetration of tissues.

5. Greater accuracy for injections where deeper tissue penetration is necessary.

6. Consistent computer controlled flow rate and pressure of LA delivery.

7. Greater ergonomic control and fixed flow rate are responsible for an improved injection experience

8. 2-3 fold reduction in pain perception

9. Least anxiety producing injection instrument based on visual appearance.

CCLAD technology has led to the development of new nerve block techniques by Friedman and Hochman: Anterior middle superior alveolar (AMSA) injection, Palatal approach anterior superior alveolar (PASA) injection

\section{Disadvantage:}

1. Requires additional armamentarium

2. Costly

3.

\section{J. Computers in Implantology}

(i) Computer Imaging Software: to simulate preoperatively the implant position into a virtual patient, that is, a patient's jaw created from the computerized tomography scan data. Computer generated surgical guides with drill holes based on presurgical virtual implant positioning. Computerassisted implant surgery (CAIS) through simultaneous tracking and guidance of the implant instrumentation. Robot-assisted surgical placement of implants is a possible future application of CAIS. ${ }^{28}$

\section{Advantages of CAIS: : $^{28}$}

1. Reduce surgical time

2. Minimize surgical invasiveness

3. Result in more precise translation on implant planning to the actual surgical procedure

4. Improved accuracy and safety

5. Surgeon validation and expertise are maintained Limitations of CAIS: ${ }^{28}$
1. Expensive

2. Increased installation time for surgery

3. Requires greatest amount of preparation and coordination of the patient, image data, and surgical instrumentation.

4. Training is mandatory

5. Inaccurate data can lead to difficulties in registration

\section{(ii) CAD/CAM in Implantology}

The CAD/CAM surgical guide fabrication revolutionizes the 3D imaging technology in the field of dentistry. The radiographic stent or a virtual restoration designed by the software helps the clinician to position the implants in the most appropriate location from a restorative standpoint. The surgical guides can be fabricated with computer assisted manufacturing (CAM) to allow for the transfer of this computer assisted design (CAD) and the 3D virtual planned treatment decisions to the actual surgical field. ${ }^{29}$ Bone dimension at the edentulous site, proximity of the planned implant site to vital anatomic structures, cost, need for sophisticated equipment and additional steps, increased accuracy and efficiency are all factors to be considered when selecting the type of surgical template for implant placement. ${ }^{31}$ Such softwares are now available from a number of manufacturers, such as Anatomage, Astra Facilitate, in 2 guide, SimPlant/Materialize, and Nobel Clinician. ${ }^{30}$

\section{Advantages: ${ }^{31}$}

1. Could offer improved precision (consistency in achieving the same implant position each time) and better accuracy (achieving desired implant location) when protocol is followed precisely, with detailed attention to every step and with appropriate patient selection,

2. Flapless surgery is possible (potential for lower morbidity

3. Efficiency (reduced surgical time)

4. Faster initial healing time (reduced trauma to soft tissues)

5. Safety (avoidance of important anatomical structures

6. Provisional restorations can be fabricated prior to the surgery

7. Could help control and maintain drill trajectory when implants are placed immediately in a fresh extraction socket.

Simplant: It is a comprehensive 3-D system for accurate and predictable implant treatment-from diagnostics to treatment planning, surgery to the final restoration. It's open platform solution is compatible with computed tomography $(\mathrm{CT})$ and cone-beam CT $(\mathrm{CBCT})$ scanners as well as intraoral scanners. With its extensive implant and abutment library, consisting of over 10,000 implants from more than 100 implant manufacturers, SIMPLANT effortlessly connects to the existing systems in which clinicians have already invested. It's restorative-driven implant treatment 
protocols allow for optimized postsurgical results with the inclusion of the proposed or final prosthesis within the planning file. The optical scan and dual scan procedures overlay the bone data from the CBCT scan together with the prosthetic information, enabling predictable, immediate provisionalization with SIMPLANT's immediate smile concept. ${ }^{32}$

\section{K. Digital Smile Design Programs}

The digital smile design (DSD) protocol allows for esthetic planning- face, smile, periodontal tissue and teeth- through the drawing of reference lines and the final dental design on extra- and intraoral digital photographs, in which the reference lines of the face and other anatomical axes are plotted as a guide to establish a proper gingival contour, shape, and dental alignment. This widens the diagnostic vision and helps the team members measure the treatment limitations and risk factors such as asymmetries, disharmonies, and violations of esthetic principles, thus provides greater predictability of treatment as it allows a final dental outline showing the relationship between the preoperative situation and the ideal design. ${ }^{33}$

Eight DSD programs are known: Photoshop CS6, keynote, aesthethic digital smile design, planmeca romexis smile designs, Cerec SW 4.2, smile design Pro, DSD app, VisagiSMile. Out of which Photoshop CS6 and keynote provides more comprehensive smile analysis whereas DSD app and smile designer pro are available as mobile phone applications. ${ }^{34}$

\section{3-D Printing in Dentistry}

The term 3D printing is generally used to describe a manufacturing approach that builds objects one layer at a time, adding multiple layers to form an object. This process is more correctly described as additive manufacturing, and is also referred to as rapid prototyping. ${ }^{35}$ The technology has a particular resonance with dentistry, and with advances in 3D imaging and modelling technologies such as cone beam computed tomography and intraoral scanning, and wit the use of CAD CAM technologies in dentistry, it will become of increasing importance. Uses of 3D printing include the production of drill guides for dental implants, the production of physical models for prosthodontics, orthodontics and surgery, the manufacture of dental, craniomaxillofacial and orthopaedic implants, and the fabrication of copings and frameworks for implant and dental restorations. ${ }^{36}$

\section{Ergonomics of Digital Dentistry}

Ergonomics can occur because of spending a lot of time sitting in front of a computer, it is important that a proper practice of body positioning and proper typing style and that a rest period is obtained at regular intervals. While sitting in front of the computer, adjust your chair so that the back is straight and neck and back muscles are relaxed. Arm supports should be adjusted so that arms form no less than a 90 degree angle at the elbow. If the back of the chair is adjustable, it should be adjusted to create gentle support for pelvis and neck. Feet should be flat on the floor, and the height of the chair should be adjusted so that thighs are approximately level with the floor. While typing, elbows should be above the level of the keyboard. This will prevent fatigue and numbing of arms that can occur from prolonged posturing of arms at an angle to the work surface. Elbows should be close to your body. Wrists should not rest on the table or keyboard when typing, as this will cause stress on wrists. Use whole arm to support your hands and fingers. While entering data or typing for a long period of time, look away from the screen for sometime and let eyes focus on a more distant object. Gently stretch and rotate neck if sitting in the same position for a long time. It may be prudent to request an ergonomically correct chair for your workstation if you cannot work with the chair you have now. ${ }^{37}$

\section{Conclusion}

The main limitation of digital dentistry is cost, to adopt new technology often requires a higher capital investment, especially at the innovator or early adopter stage. Misunderstanding the new technology tends to foster slower adoption rates. This scenario can be easily avoided by greater attendance of basic \& advanced hands-on courses in these areas of technology. In the coming years there will be a further huge flood of new improvements and introduction of new materials and devices. However, the digital technologies have already begun to dramatically change the world of dentistry hence the future is NOW!

\section{References}

1. Guynup S. How Technology is Changing Dentistry [Internet]. Scientific American Inc 2016. Available from: https://www.scientificamerican.com/products/the-futureof-oral-health/how-technology-is-changing-dentistry/.

2. Jevremovic DP, Puskar TM, Budak I, Vukelic D, Kojic $\mathrm{V}$, Eggbeer D, et al. An RE/RM approach to the design and manufacture of removable partial dentures with a biocompatibility analysis of the F75 Co-Cr SLM alloy. Materiali in Tehnologije 2012;46 (2):123-9.

3. Collen MF. A history of medical informatics in the United States. Washington, DC: American Medical Informatics Association Springer; 1995.

4. Schleyer TK. Dental informatics: an emerging biomedical informatics discipline. J Dent Educ. 2003;67(11):1193200.

5. Soffar H. Computer technology in dentistry (Digital dentistry) uses, types, cons \& pros. [Internet] 2017. Available from: www.online-sciences.com.

6. Sandoval VA, Dale RA, Hendricson WD, Alexander JB. A comparison of four simulation and instructional methods for endodontic review. J Dent Educ 1987;51:532-8.

7. Tira DE. Evaluation of a CAI course in a removable partial prosthodontics classification system. J Comput Based Instruct 1977;4:34-42. 
8. Advantages, disadvantages of electronic medical records. [Internet] 2009. Available from:

http://m.bctv.org/mobile/special_reports/health/advantage s-disadvantages-of-electronic-medical-records/article.

9. Schleyer T, Song M, Gregg H. Gilbert D, Rindal B, Jeffrey L, Valeria V, Ellen F. Electronic dental record use and clinical information management patterns among practitioner-investigators in The Dental Practice-Based Research Network. J Am Dent Assoc. 2013;144(1):4958.

10. Rawson RD. Computers in forensic dentistry. J Calif Dent Assoc 1996; 24(5):58-61.

11. Patel J, Singh HP, Paresh M, Verma C. Forensic odontology in the era of computer and technology. Int $\mathrm{J}$ Med Dent Sci 2013;2(1):59-64.

12. Buchanan H. Development of a computerized dental anxiety scale for children: validation and reliability. $\mathrm{Br}$ Dent J 2005;199(6):359-62.

13. Nelson L J, Cooper J. Gender differences in children's reactions to success and failure with computers. Computers in Human Behaviour 1997;13:247-67.

14. Ali Z and Bhaskar SB. Basic statistical tools in research and data analysis. Ind J Anaesth. 2016;60(9):662-69.

15. Samaras C. Intraoral cameras: the value is clear. Compend Contin Educ Dent 2005;26:456-58.

16. Magnusson I. Computerized periodontal probing. Periodontol 2000 1996;12:40-3.

17. Paul L. Digital dentistry: Is this the future of dentistry? [Internet]. Child Jr 2011. Available from: http://www.dentaleconomics.com.

18. Bragger U. Radiographic parameters: biological significance and clinical use. Periodontol 2000 2005;39:73 - 90 .

19. Sainu R J. Imaging Techniques in Periodontics: A Review Article. Biosci Tech 2016;7(2):739-47.

20. Brennan. An introduction to digital radiography in dentistry. J Orthodontics 2002;29:66-9.

21. Parks ET et al. Digital Radiography: An overview. J Contemp Dent Pract 2002;4 (3):23-39.

22. Akarslan $\mathrm{ZZ}$ et al. Advances in radiographic techniques used in dentistry. Turkey : "Emerging Trends in Oral Health Sciences and Dentistry"2015;(34) 763-788.

23. Vos W D et al. Cone beam computerized tomography imaging of the oral and maxillofacial region:A systematic review of the literature. Int J. Oral Maxillofac Surg 2009;38:609-25.

24. Hsieh YS, Ho YC, Lee SY, Chuang CC, Tsai JC, Lin KF and Sun CW. Dental Optical Coherence Tomography. Sensors 2013;13:8928-49.

25. Rajaa M, Mussawi AA, Farid F. Computer-Based Technologies in Dentistry: Types and Applications. J of Dent 2016; 13(3):215-22.

26. Kerstein RB. Current applications of computerized occlusal analysis in dental medicine. Gen Dent. 2001 ;49(5):521-30.

27. Kwak EJ, Pang NS, Cho JH, Jung BY, Kim KD, Park W. Computer-controlled local anesthetic delivery for painless anesthesia: a literature review.. J Dent Anesth Pain Med 2016;16(2):81-88.

28. Abbas A \& Sakineh N. Computer-assisted implantology: Historical background and potential outcomes - A review. The international journal of medical robotics + computer assisted surgery 2008;4:95-104.

29. Jung RE, Schneider D, Ganeles J, et al. Computer technology applications in surgical implant dentistry: a systematic review. Int J Oral Maxillofac Implants 2009;24:92-109.
30. Alzoubi F, Massoomi N, Nattestad A. Accuracy of Immediate and Delayed Placements Using CAD/CAM Guides. J Oral Implantol 2016;42(5):391-98.

31. Kattadiyil MT, Parciak E, Puri S, Scherer MD. $\mathrm{CAD} / \mathrm{CAM}$ guided surgery in implant dentistry: a brief review. Alpha Omegan. 2014 Spring;107(1):26-31.

32. SIMPLANT Computer-Guided Implant Treatment Software: Unlocking Digital Potential. Compend Contin Educ Dent. 2014;35(10):782.

33. Coachman C, \& Calamita M. Digital smile design: A tool for treatment planning and communication in esthetic dentistry. Quintessence of Dental Technology 2012;35:103-111.

34. Omar D.The application of parameters for comprehensive smile esthetics by digital smile design programs: A review of literature. Saudi Dent J 2018; 30(1):7-12.

35. AndonoviĆ V, Vrtanoski G. Growing rapid prototyping as a technology in dental medicine. Mech Eng Sci J 2010; 29:31-39.

36. Dawood A, Marti Marti B, Sauret-Jackson V and Darwoo A. 3D printing in dentistry. Br. Dent J 2015;219(11):52129.

37. Computer Ergonomics: How to Protect Yourself from Strain and Pain. [Internet]. https://www.uhs.umich.edu/computerergonomics.

38. Kumar P, Kumar P. Modern Dentistry @Computerization Dot Com; An epigrammatic sketch of the present scenario. J Adv Med Dent Scie Res 2013;1(1):17-28. 